\title{
Determinants of fathers' involvement in breastfeeding practices in Kuala Selangor
}

\author{
Nurul Izyan Mat Nawi ${ }^{1}$ \& Syahrul Bariah Abdul Hamid ${ }^{2}$ \\ ${ }^{1}$ Centre of Nutrition and Dietetics, Faculty of Health Sciences, Universiti Teknologi \\ MARA, Puncak Alam Campus, 42300 Puncak Alam, Selangor, Malaysia; ${ }^{2}$ Mother, \\ Infant and Young Child Nutrition (MiChild) Research Group, Faculty of Health \\ Sciences, Universiti Teknologi MARA, Puncak Alam Campus, 42300 Puncak Alam, \\ Selangor, Malaysia
}

\begin{abstract}
Introduction: Fathers' knowledge, attitude, and involvement in breastfeeding practices are crucial factors to determine the success of breastfeeding outcomes such as decision-making on breastfeeding, successfulness of exclusive breastfeeding, and continuation of breastfeeding practices. This study aimed to identify the determinants of fathers' involvement in breastfeeding practices. Methods: This cross-sectional study involved 203 fathers attending Maternal and Child Health Clinics in Kuala Selangor. Self-administered questionnaires were used to obtain information regarding fathers' sociodemography, breastfeeding knowledge, attitudes, and involvement towards breastfeeding practices. Results: Mean age of the fathers was $32.1 \pm 5.9$ years, ranging between 21 to 50 years. Findings showed that majority of the fathers were involved in breastfeeding practices $(52.7 \% ; n=107$, $57.0 \pm 6.2)$, had fair knowledge in breastfeeding $(62.6 \% ; n=127,13.8 \pm 3.2)$, and showed low attitude towards breastfeeding $(68.0 \% ; n=138,61.9 \pm 6.7)$. Multiple logistic regression analysis revealed factors with significant influence on fathers' involvement towards breastfeeding practices were: (1) fathers' breastfeeding knowledge, (2) fathers' attitudes towards breastfeeding practices, and (3) mode of infant delivery. Conclusion: These significant predictors may help healthcare professionals to establish effective strategies by advocating the role of fathers in the breastfeeding process such as by offering continuous support, active involvement, and effective participation.
\end{abstract}

Keywords: Breastfeeding support, paternal involvement, breastfeeding knowledge, breastfeeding attitude, involvement

\section{INTRODUCTION}

The World Health Organization (WHO) has highlighted the importance of exclusive breastfeeding among mothers for the first six months of their infant's life to achieve optimal health, growth, and development. The Academy of Nutrition and Dietetics has stated that breastfeeding with complementary foods from six months to at least twelve months of age is the ideal feeding pattern for infants (Lessen \& Kavanagh, 2015). Breastfeeding provides many nutritional benefits, is convenient and inexpensive as compared to formula milk. According to the Fourth National Health and Morbidity Survey (IPH, 2016), 65.3\% of

\footnotetext{
*Corresponding author: Dr. Syahrul Bariah Abdul Hamid

Centre of Nutrition and Dietetics, Faculty of Health Sciences, Universiti Teknologi MARA,

Puncak Alam Campus, 42300 Puncak Alam, Selangor, Malaysia

Tel: (6)(03)32584382; Fax: (6)(03) 32584599; E-mail: syahrulbariah@uitm.edu.my

doi: https://doi.org/10.31246/mjn-2020-0070
} 
infants under six months of age were breastfed within the first hour of delivery, $47.1 \%$ of infants under six months of age were breastfed exclusively, 39.4\% of infants between 20 to 23 months of age continued on breastfeeding. Overall, the prevalence of breastfeeding practices in the latest survey is increasing as compared to the survey conducted in 2006, but it still places Malaysia with the lowest prevalence of exclusive breastfeeding practices as compared to other Southeast Asian countries. Moreover, the overall prevalence of exclusive breastfeeding has not met the target level of $70 \%$, which is to be achieved by 2025 (WHO \& UNICEF, 2014 \& NCCFN, 2016).

The decision on breastfeeding is usually influenced by many factors. Factors influencing breastfeeding includes the provision of support from family members and healthcare providers (Draman et al., 2017; Leahywarren et al., 2014), level of education (Laanterä, Pietilä, \& Pölkki, 2010; Leahywarren et al., 2014), previously having breastfed or first-time mothers (Leahywarren et al., 2014), having a positive attitude towards breastfeeding and high breastfeeding self-efficacy (Lester, 2014; Abdul Hamid \& Yahya, 2018), working mothers (Amin et al., 2011), and mothers facing breastfeeding difficulties (Hobbs et al., 2016).

A recent study has shown that fathers play an important role that contributes to the success of breastfeeding outcomes. Sherriff, Hall, \& Panton (2014) revealed that fathers' breastfeeding knowledge, positive attitude towards breastfeeding, participation in decision-making in breastfeeding, provision of practical support, and emotional support were five key attributions of fathers' role in breastfeeding. Previous studies have shown increasing evidence that fathers may influence breastfeeding outcomes such as decision-making in breastfeeding (dos Santos et al., 2016; Draman et al., 2017; de Montigny et al., 2018; Rempel, Rempel \& Moore, 2017), early initiation of breastfeeding, the successfulness of exclusive breastfeeding (Draman et al., 2017; Phua, Razak \& Shukri, 2020), and continuation of breastfeeding (de Montigny et al., 2018).

Mothers are pleased to have fathers' support, active involvement and effective participation in breastfeeding practices (dos Santos et al., 2016). Mothers consider fathers play an important role in the breastfeeding period by offering emotional and physical support, which are critical components of breastfeeding success, especially when mothers encounter feeding difficulties. According to Binns et al. (2009), the provision of practical and emotional support from fathers is a key factor for successful breastfeeding, increasing the confidence of mothers and enabling them to maintain sufficient milk production. Besides, a study conducted by Draman et al. (2017) showed that fathers' decision and motivation for breastfeeding has a positive effect on exclusive breastfeeding practices among mothers.

To the best of our knowledge, the study on fathers' role in breastfeeding is very limited in Malaysia. To date, only three studies have been conducted to investigate father's influence towards breastfeeding. Phua and colleagues found that first time mothers with good paternal support had longer breastfeeding duration (Phua et al., 2020), meanwhile another study discovered that there was no difference in breastfeeding knowledge and attitude among fathers in the exclusive and non-exclusive breastfeeding groups (Mohamad et al., 2015). However, Draman and colleagues suggested that mutual decision on breastfeeding practices imposed a greater influence towards exclusive breastfeeding practices (Draman et al., 2017). 
Therefore, there is a need to identify the determinants of fathers' involvement in breastfeeding. Understanding the determinants of fathers' involvement in breastfeeding practices may assist health professionals and policy makers to provide significant strategies focusing on the promotion of breastfeeding, directly steering fathers as breastfeeding supporters, particularly in exclusive breastfeeding practices for the first six months of life. Therefore, this study aims to identify the determinants of fathers' involvement in breastfeeding practices among fathers attending Kuala Selangor and Bestari Jaya primary health care facilities in Selangor, Malaysia.

\section{MATERIALS AND METHODS}

\section{Subjects and study design}

This was a cross-sectional study involving fathers accompanying their wives whom were attending the Kuala Selangor and Bestari Jaya maternal and child primary healthcare facilities in Selangor, Malaysia. Sample size calculation was made by using G* Power Software. Total sample size calculated by the software required 170 fathers in this study, based on power 0.9 , type 1 error 0.05 and effect size 0.22 by a previous study (Mitchell-Box et al., 2013). A total of 203 fathers were recruited and selected by convenience sampling. The inclusion criteria were biological fathers of a fullterm, healthy singleton baby aged two years and below, and have literacy in either the Malay or English language. Illiterate fathers, diagnosed with psychiatric disorders, and foreigners were excluded from the study.

\section{Data collection}

Data were collected from September 2019 to October 2019. Each father was given a set of self-administered questionnaires consisting of sixty-eight questions divided into five sections (A,
B, C, D, and E). Section A included nine questions to obtain the participants' demographic information. These included the fathers' age, ethnicity, level of education, occupation, income, parity, and participation in antenatal and breastfeeding classes. Section $B$ contained six questions to gather information about infants. Section C contained twenty-two questions used to elicit information on breastfeeding knowledge of the respondents. Section $\mathrm{D}$ included seventeen questions that covered fathers' attitudes towards breastfeeding. Lastly, section E contained fourteen questions used to elicit information on fathers' involvement during the breastfeeding period. Fathers were given sufficient time to complete the questionnaire. Once completed, the questionnaires were reviewed for completion by the researchers.

\section{Breastfeeding knowledge scale}

The scale was developed by Laanterä, Pietilä \& Pölkki in 2010, and has good internal consistency reliability measured by Cronbach's alpha coefficient, which is 0.84 . The scale was used to measure participants' knowledge regarding breastfeeding in the hospital and at home, breastfeeding management, lactation, and specific situations such as breast problems (Laanterä et al., 2010). This scale consisted of 22 items in which a four-point Likert scale ranging from "strongly agree" to "strongly disagree" and two openended questions were used. All the statements were reclassified, whereby the "strongly agree" and "somewhat agree" responses were combined to form "agree", while the "somewhat disagree" and "strongly disagree" responses were combined to form "disagree". Likert scale statements and open-ended questions were measured by awarding one point for each correct answer and zero if the answer was incorrect or incomplete. 
The question regarding breastfeeding benefits was assessed by awarding one point if at least one breastfeeding benefit was reported and the question on how to increase lactation was assessed by awarding one point if increasing breastfeeding frequency or pumping or breastfeeding at night was suggested. A maximum of 22 points could be obtained by the fathers in the breastfeeding knowledge test.

\section{Iowa Infant Feeding Attitude Scale (IIFAS)}

The IIFAS was used to measure the attitudes of fathers towards breastfeeding practices. It has an internal consistency reliability measured by Cronbach's alpha coefficient that ranges between 0.78 and 0.85 in most studies (de la Mora et al., 2006). The IIFAS have been translated and validated in the Malay language and has been used among mothers in Malaysia (Shukri et al., 2017). The IIFAS consisted of 17 questions, eight questions were favourable towards breastfeeding, while nine questions favourable towards formula feeding. Fathers were asked to indicate the extent to which they agree with each statement, on a five-point Likert scale ranging from "strongly disagree" to "strongly agree." Items that were asterisked were reversedly scored $(1=5,2=4,4=2,5=1)$ (de la Mora et al., 2006). Total attitude score could range from 17 to 85, with higher scores reflecting a more positive attitude towards breastfeeding. Previous researchers have grouped total score into the following five categories: (1) very positive towards breastfeeding (8185), (2) positive towards breastfeeding (70-80), (3) neutral (49-69), (4) positive towards formula feeding (38-48), and (5) very positive towards formula feeding (17-37). However, in this study, a score of $>65$ was considered a positive attitude towards breastfeeding (Cox, Giglia \& Binns, 2015).

\section{Fathers' involvement in breastfeeding scale}

The scale was developed by Abu-Abbas, Kassab \& Shelash in 2016 to measure fathers' involvement in breastfeeding practices. It consisted of 14 items with a five-point Likert scale ranging from "strongly disagree" to "strongly agree", with a Cronbach's alpha of 0.73. This questionnaire has good internal consistency and reliability (Abu-Abbas, Kassab \& Shelash, 2016). The total possible score for the questionnaire was 70 and scores of $\geq 58$ suggested good involvement in breastfeeding practices, while scores $<58$ indicated poor involvement.

\section{Data analysis}

SPSS version 21.0 was used to analyse the data. Mean scores and standard deviation were calculated to measure fathers' knowledge, attitudes, and involvement in breastfeeding practices. Simple logistic regression analysis was used to determine the possible predictive variables affecting fathers' level of involvement in breastfeeding before conducting multiple logistic regression. To restrict the number of potential predictors in multiple logistic regression analysis, the results from simple logistic regression were examined and potential predictors were retained only if $p$-value was <0.25. Multiple logistic regression was used to identify the determinants of fathers' involvement in breastfeeding. Statistical significance was set at $p<0.05$. Data were presented in the form of tables and figures.

\section{Ethical approval}

Written informed consent was obtained from the participants to ensure privacy. The questionnaires were selfadministered and data were collected anonymously. The study was approved by the UiTM Research Ethics Committee (REC/151/17) and Medical Research 
Ethics Committee, Ministry of Health (NMRR-19-2649-49971).

\section{RESULTS}

\section{Demographic variables}

A total of 203 fathers were recruited in this study. Table 1 shows the characteristics of fathers. The age of the fathers in this study ranged between 21-50 years old with a mean age of $32.14 \pm 5.91$ years old. Majority of the fathers $(83.3 \%)$ were Malays. About
$98.5 \%$ of the fathers indicated that they were living with their partners. Over half of the fathers did not pursue higher education (54.2\%). Majority of the fathers $(68.5 \%)$ had a monthly income of <RM3000. Only $37.4 \%$ of fathers were first-time fathers. Besides, majority of fathers were never involved in antenatal $(88.7 \%)$ and breastfeeding classes $(85.7 \%)$. Meanwhile, the age of the infants ranged between 2 days to 24 months. Most of the infants (72.9\%)

Table 1. Characteristics of participants $(N=203)$

\begin{tabular}{|c|c|c|c|}
\hline Variable & $M \pm S D$ & $n$ & $\%$ \\
\hline \multicolumn{4}{|l|}{ Clinic } \\
\hline KK Kuala Selangor & & 143 & 70.4 \\
\hline KK Bestari Jaya & & 60 & 29.6 \\
\hline \multicolumn{4}{|l|}{ Age } \\
\hline$<30$ years old & $32.1 \pm 5.9$ & 83 & 40.9 \\
\hline$\geq 30$ years old & & 120 & 59.1 \\
\hline \multicolumn{4}{|l|}{ Ethnicity } \\
\hline Malay & & 169 & 83.3 \\
\hline Others & & 34 & 16.7 \\
\hline \multicolumn{4}{|l|}{ Living } \\
\hline With partner & & 200 & 98.5 \\
\hline Without partner & & 3 & 1.5 \\
\hline \multicolumn{4}{|l|}{ Education level } \\
\hline Secondary school or lower & & 114 & 54.2 \\
\hline Higher than secondary school & & 89 & 45.8 \\
\hline \multicolumn{4}{|l|}{ Monthly income } \\
\hline$\leq \mathrm{RM} 3000$ & & 139 & 68.5 \\
\hline$\geq \mathrm{RM} 3001$ & & 64 & 31.5 \\
\hline \multicolumn{4}{|l|}{ Number of children } \\
\hline First child & & 76 & 37.4 \\
\hline Not first child & & 127 & 62.6 \\
\hline \multicolumn{4}{|l|}{ Involved in antenatal class } \\
\hline Yes & & 23 & 11.3 \\
\hline No & & 180 & 88.7 \\
\hline \multicolumn{4}{|l|}{ Involved in breastfeeding class } \\
\hline Yes & & 29 & 14.3 \\
\hline No & & 174 & 85.7 \\
\hline
\end{tabular}


Table 2. Level of knowledge, attitude and involvement in breastfeeding among participants $(N=203)$

\begin{tabular}{lcc}
\hline Variables & Mean & SD \\
\hline Father's knowledge in breastfeeding & 13.8 & 3.2 \\
Father's attitude in infant feeding & 61.9 & 6.7 \\
Father's involvement in breastfeeding & 57.0 & 6.2 \\
\hline
\end{tabular}

were delivered through vaginal delivery, but $5.4 \%$ of them were through assisted vaginal delivery. Majority of the infants were ever breastfed $(96.6 \%)$ and still being breastfeed (67.0\%). Forty-six infants stopped exclusive breastfeeding within the first three months $(22.7 \%)$.

Table 2 depicts the scores of breastfeeding knowledge, infant feeding attitudes, and involvement in breastfeeding among fathers.

\section{Level of breastfeeding knowledge among fathers}

The knowledge scores of the study population ranged from $5-20$ points out of the possible 22 , with a mean score of $13.8 \pm 3.2$ and $62.6 \%$ of fathers correctly answered the breastfeeding knowledge questionnaire. About one quarter of the fathers knew that the consistency of breast milk and formula is equal $(23.2 \%$, $n=47$ ).

Majority of the fathers knew that it is recommended to breastfeed a healthy infant regularly (93.6\%, $n=190)$. More than half of the fathers expressed that infant gets milk from bottle similar to breastfeeding $(58.6 \%$, $n=119$ ). Only $34 \%$ fathers believed that most mothers with small breasts have insufficient lactation $(n=69)$. Meanwhile, $44.8 \%$ knew that the nipples will be cracked if breastfeeding lasts over 10 minutes $(n=91)$. Approximately half of the fathers believed that cracked nipples happened because the infant is allowed to latch too long in the first days of life $(49.8 \%, n=101)$. A large number of fathers correctly answered that a dummy should not be used to prevent cracked nipples $(68.5 \%, n=139)$ and donor milk is not used in the making of formula $(83.3 \%, n=169)$. About $45.3 \%$ of the fathers believed that there is a need to give water to all infants, including exclusively breastfed infants, especially on hot days.

More than half of the fathers believed that it is recommended to follow a special time schedule while breastfeeding (69.5\%, $n=141)$. Only $29.6 \%$ fathers thought that breastfed infants need complementary foods from at least 4 months of age $(n=60)$. A total of $42.9 \%$ fathers reported that it is recommended to cease breastfeeding if a breastfeeding mother has diarrhoea $(n=87)$, while 90 fathers wrongly answered on the item "it is recommended to pump the breasts after alcohol consumption before the next breastfeeding" (44.3\%). A large proportion of the fathers correctly answered on the item "breastfed or formula-fed infants have as many ear infections until they are 1 year old" (86.7\%, $n=176)$.

\section{Level of fathers' attitudes in breastfeeding}

Level of breastfeeding attitude among fathers was measured using IIFAS. The scores of fathers' attitudes in breastfeeding ranged from 43 to 77 points out of the possible 85 , with a mean score of $61.9 \pm 6.7$. A score of >65 was considered a positive attitude towards breastfeeding (Cox, Giglia, $\&$ Binns, 2015). More than half of the fathers $(68 \%, n=138)$ were found to have 
a lower breastfeeding attitude, whom had more positive attitudes towards formula feeding compared to fathers who had positive attitudes towards breastfeeding (32\%, $n=65)$.

The lowest mean score was 2.46 from the item "a mother who occasionally drinks should not breastfeed her baby", which reflected a negative attitude in breastfeeding. The highest mean score was 4.64 on the item "breast milk is the ideal food for babies", where majority of fathers managed to answer correctly. Almost half of the fathers agreed that the nutritional benefits of breast milk last only until the baby is weaned from breast milk (46.8\%, $n=95)$. Majority of fathers disagreed that formula feeding is more convenient than breastfeeding (50.7\%, $n=103)$. A large number of fathers managed to answer correctly on the item "breast milk is lacking in iron" (71.5\%, $n=145)$.

More than half of the fathers agreed that formula feeding is a better choice if a mother plans to work outside of home (53.7\%, $n=109)$. Majority disagreed that women should not breastfeed in public places such as restaurants (55.2\%, $n=112)$. Approximately half of the fathers thought that breastfed babies are more likely to be overfed than formula-fed babies $(53.2 \%, n=108)$. Most of the fathers disagreed that formula milk is as healthy as breast milk for an infant $(68 \%, n=138)$. Over half of the fathers wrongly answered on the item "a mother who occasionally drinks should not breastfeed her baby" (52.2\%, $n=106)$.

\section{Level of father's involvement in breastfeeding}

Meanwhile, the scores of fathers' involvement in breastfeeding ranged from 39 to 70 points, with a mean score

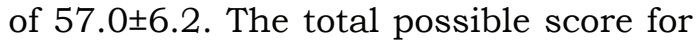
the questionnaire was 70 and a score of $\geq 58$ indicated good involvement in the breastfeeding process, while a score of $<58$ indicated poor involvement (AbuAbbas et al., 2016). A total of 107 $(52.7 \%)$ fathers were found to have good involvement in breastfeeding and the rest represented poor involvement in breastfeeding $(47.3 \%, n=96)$.

The mean score of the item "showed acceptance regarding formula feeding" was 2.76 , which was the least among all involvement items, reflecting a negative involvement in breastfeeding. Meanwhile, the item "helped your wife with houseworks and taking care of other baby tasks like responding to the baby's cries, help in bathing the baby... etc." was 4.58, which was the highest score, reflecting a positive involvement in breastfeeding. Majority of the fathers agreed with their wife's desire to stop breastfeeding $(40.4 \%, n=82)$. A total of $43.9 \%$ of fathers showed acceptance regarding formula feeding $(n=89)$. A large number of fathers admitted not getting upset if other houseworks were not done during the breastfeeding period (69.9\%, $n=142$ ).

\section{Determinants of fathers' involvement in breastfeeding}

Table 3 shows the results of Simple Logistic Regression (SLR) analysis. SLR was done to identify the possible predictors affecting fathers' involvement in breastfeeding practices before running the Multiple Logistic Regression (MLR) analysis. As shown in Table 3, there were significantly associated factors for fathers' involvement in breastfeeding practices: knowledge and attitude $(p<0.001)$. Only ethnicity, education, occupation, income, baby's age, mode of delivery, knowledge and attitude scores were selected to be included in the MLR analysis as they had a $p$-value of $<0.25$.

Table 4 shows the results of MLR analysis to identify the determinants of fathers' involvement in breastfeeding 
Table 3. Factors associated with fathers' involvement in breastfeeding practices using simple logistic regression

\begin{tabular}{|c|c|c|c|c|c|c|}
\hline \multirow[t]{2}{*}{ Variables } & \multicolumn{2}{|c|}{$\begin{array}{c}\text { Good } \\
\text { involvement } \\
(n=96)\end{array}$} & \multicolumn{2}{|c|}{$\begin{array}{c}\text { Poor } \\
\text { involvement } \\
(n=107)\end{array}$} & \multirow[t]{2}{*}{ Crude OR (95\% CI) } & \multirow[t]{2}{*}{ p-value } \\
\hline & $n$ & $\%$ & $n$ & $\%$ & & \\
\hline \multicolumn{7}{|l|}{ Ethnicity } \\
\hline Malay & 83 & 86.5 & 86 & 80.4 & $1.56(0.77,3.32)$ & \multirow{2}{*}{$0.249^{*}$} \\
\hline Others & 13 & 13.5 & 21 & 19.6 & 1.00 & \\
\hline \multicolumn{7}{|l|}{ Education } \\
\hline $\begin{array}{l}\text { Higher than secondary } \\
\text { schools }\end{array}$ & 49 & 51.0 & 40 & 37.4 & $1.75(1.00,3.06)$ & \multirow{2}{*}{$0.051^{*}$} \\
\hline $\begin{array}{l}\text { Secondary school and } \\
\text { lower }\end{array}$ & 47 & 49.0 & 67 & 62.6 & 1.00 & \\
\hline \multicolumn{7}{|l|}{ Occupation } \\
\hline Private/public sectors & 73 & 76.0 & 68 & 63.6 & $1.82(0.99,3.36)$ & \multirow[b]{2}{*}{$0.055^{*}$} \\
\hline $\begin{array}{l}\text { Self-employed, not } \\
\text { working }\end{array}$ & 23 & 24.0 & 39 & 36.4 & 1.00 & \\
\hline \multicolumn{7}{|l|}{ Income } \\
\hline$\geq$ RM3001 & 36 & 37.5 & 28 & 26.2 & $1.69(0.93,3.08)$ & \multirow{2}{*}{$0.084 *$} \\
\hline$\leq \mathrm{RM} 3000$ & 60 & 62.5 & 79 & 73.8 & 1.00 & \\
\hline \multicolumn{7}{|l|}{ Parity } \\
\hline Primiparous & 37 & 38.5 & 39 & 36.4 & $1.09(0.62,1.93)$ & \multirow{2}{*}{0.758} \\
\hline Multiparous & 59 & 61.5 & 68 & 63.6 & 1.00 & \\
\hline \multicolumn{7}{|l|}{$\begin{array}{l}\text { Attendance to } \\
\text { antenatal class }\end{array}$} \\
\hline Yes & 12 & 12.5 & 11 & 10.3 & $1.25(0.52,2.97)$ & \multirow{2}{*}{0.619} \\
\hline No & 84 & 87.5 & 96 & 89.7 & 1.00 & \\
\hline \multicolumn{7}{|l|}{$\begin{array}{l}\text { Attendance to } \\
\text { breastfeeding class }\end{array}$} \\
\hline Yes & 15 & 15.6 & 14 & 13.1 & $1.23(0.56,2.70)$ & \multirow{2}{*}{0.606} \\
\hline No & 81 & 84.4 & 93 & 86.9 & 1.00 & \\
\hline \multicolumn{7}{|l|}{ Baby's age } \\
\hline$\leq 6$ Months & 45 & 46.9 & 60 & 56.1 & $0.69(0.40,1.20)$ & \multirow{2}{*}{$0.191^{*}$} \\
\hline$\geq 7$ Months & 51 & 53.1 & 47 & 43.9 & 1.00 & \\
\hline \multicolumn{7}{|l|}{ Mode of delivery } \\
\hline Normal & 76 & 79.2 & 72 & 67.3 & $1.85(0.98,3.49)$ & \multirow{2}{*}{$0.059 *$} \\
\hline Caesarean & 20 & 20.8 & 35 & 32.7 & 1.00 & \\
\hline Fathers' age, $n=203$ & & & & & $1.00(0.95,1.04)$ & 0.844 \\
\hline $\begin{array}{l}\text { Breastfeeding Knowledge } \\
\text { Score, } n=203\end{array}$ & & & & & $1.29(1.17,1.44)$ & $<0.001^{*}$ \\
\hline $\begin{array}{l}\text { IIFAS Score (Attitude), } \\
n=203\end{array}$ & & & & & $1.17(1.11,1.23)$ & $<0.001^{*}$ \\
\hline
\end{tabular}


Table 4. Factors associated with fathers' involvement in breastfeeding practices using multiple logistic regression analysis

\begin{tabular}{|c|c|c|c|c|c|}
\hline Variables & $n$ & $\begin{array}{c}\text { Crude OR } \\
\text { (95\% CI) }\end{array}$ & p-value & $\begin{array}{c}\text { Adjusted OR } a \\
\quad(95 \% \text { CI) }\end{array}$ & p-value \\
\hline \multicolumn{6}{|c|}{ Mode of delivery } \\
\hline Normal & 148 & $1.85(0.98,3.49)$ & \multirow{2}{*}{0.059} & $2.12(1.03,4.36)$ & \multirow{2}{*}{$0.040^{*}$} \\
\hline Caesarean & 55 & 1.00 & & 1.00 & \\
\hline Knowledge & 203 & $1.29(1.17,1.44)$ & $<0.001^{*}$ & $1.17(1.04,1.31)$ & $0.008^{* *}$ \\
\hline Attitude & 203 & $1.17(1.11,1.23)$ & $<0.001^{*}$ & $1.14(1.07,1.20)$ & $<0.001^{*}$ \\
\hline
\end{tabular}

${ }^{a} \mathrm{~A}$ forward LR and backward LR multiple logistics regression model was applied ${ }^{*} p<0.05,{ }^{* *} p<0.01$

practices. Crude and adjusted odds ratios of variables associated with fathers' involvement in breastfeeding are shown in Table 3. Fathers who had a baby born through vaginal delivery were 2.1 times more likely to have good involvement in breastfeeding practices than fathers who had a baby delivered through C-section (crude OR: 1.845, 95\% CI: 0.98 - 3.49, adjusted OR: $2.12,95 \%$ CI of adjusted OR: $1.03-$ 4.36). Besides, fathers with a higher score in breastfeeding knowledge were more likely to have good involvement in breastfeeding practices than fathers with a lower score (crude OR: 1.29, 95\% CI of crude OR: $1.17,1.44)$, and the adjusted odds ratio was 1.17 (95\% CI of adjusted OR: 1.04 - 1.31). As expected, fathers with a higher score in attitude towards breastfeeding were more likely to have good involvement in breastfeeding practices than fathers with a lower score (crude OR: $1.17,95 \% \mathrm{CI}$ of crude OR: $1.11,1.23)$, and the adjusted odds ratio was 1.14 (95\% CI of adjusted OR: 1.071.20).

Ethnicity, education level, working status, monthly income, age of infant were not significant predictors influencing fathers' involvement in breastfeeding. The following demographic variables were also not significant factors affecting fathers' involvement in breastfeeding practices: total number of children, involvement in antenatal class, involvement in breastfeeding class, age of the infant, infant ever being breastfed, and duration exclusive breastfeed.

\section{DISCUSSION}

\section{Determinants of fathers' involvement in breastfeeding}

The present study found that fathers whose baby was delivered vaginally were 2.1 times more likely to have good involvement in breastfeeding practices than fathers whose baby was delivered through $\mathrm{C}$-section. These findings may be related to the early cessation of breastfeeding by mothers who undergo caesarean delivery. In one prospective cohort study in Calgary, Alberta involving 3021 mothers found that more mothers who delivered by planned $\mathrm{C}$-section had no intention to breastfeed or did not initiate breastfeeding when compared to women with vaginal births (Hobbs et al., 2016). Therefore, they were more likely to cease breastfeeding before 12 weeks postpartum compared to those who delivered vaginally. Also, according to one meta-analysis study conducted by Zhao et al., (2017) in China, in the early postpartum period, the odds of exclusive breastfeeding after a caesarean section was $47 \%$ lower than after vaginal delivery. These findings from previous studies showed that breastfeeding practices were affected adversely by 
caesarean delivery. Given that mothers who had caesarean delivery will be more likely to have adverse breastfeeding outcomes, therefore it is suggested for fathers to continuously support their spouses to initiate breastfeeding and continue to breastfeed. Moreover, it is important to advocate future fathers on the importance of breastfeeding and how they could play their roles as a supportive partner especially during postpartum period.

From the present study, it was shown that fathers with a higher score in breastfeeding knowledge were more likely to have good involvement in breastfeeding practices than fathers with a lower score. In one crosssectional study conducted by Abhinaya, Arunprasath \& Padmasani (2016) in India among 93 fathers, they found that fathers with good knowledge had a positive attitude towards breastfeeding. In that study, fathers' breastfeeding knowledge was positively influenced by healthcare exposure and education (Abhinaya et al., 2016). Leng et al. (2019) found that fathers' breastfeeding knowledge was the second predictor that influenced fathers' involvement in breastfeeding. A father who has good breastfeeding knowledge can improve breastfeeding continuity (Maycock et al., 2015; Sherriff et al., 2014). According to one community-based intervention study in Vietnam conducted by Bich \& Cuong (2016), fathers' knowledge regarding breastfeeding was shown as a firm foundation for fathers' attitudes and involvement in breastfeeding. Fathers who have limited breastfeeding knowledge reported that they would like to gain more information regarding breastfeeding (de Montigny et al., 2018; $\mathrm{Ng}$, Shorey \& He, 2019).

Besides that, fathers with a higher score in attitude towards breastfeeding were more likely to have good involvement in breastfeeding practices than fathers with a lower score. Fathers' attitudes towards breastfeeding are associated with fathers' involvement in the breastfeeding process (Lester, 2014; Rempel et al., 2017), as they influence the initiation and success of breastfeeding ( $\mathrm{Ng}$ et al., 2019). In one correlational study conducted by Leng et al. (2019) in Singapore, the perceived acceptance of fathers by family members and friends, fathers' knowledge about breastfeeding, perceived behaviour control, marital satisfaction, and perceived improvements of knowledge and attitudes during two weeks postpartum were significant factors affecting fathers' involvement in breastfeeding practices. According to one integrative review study conducted by $(\mathrm{Ng}$ et al. (2019), factors that may influence fathers' involvement in breastfeeding included fathers' knowledge regarding breastfeeding, fathers' attitudes towards breastfeeding, fathers' perceptions of subjective norms surrounding their involvement in breastfeeding, fathers' perceived behavioural control of their involvement, committed relationship, socio-demographic characteristics, and fathers' attendance at antenatal classes. These findings showed that both fathers' knowledge and attitudes in breastfeeding can influence fathers' involvement during the breastfeeding period. Higher levels of paternal support and encouragement are linked with greater maternal confidence to breastfeed, as fathers are the main source of social support for mothers who are breastfeeding (Al-Namir, Brady \& Gallagher, 2017). According to Al-Namir et al. (2017), excluding fathers from breastfeeding education, support and training can result in lower quality of life and father self-efficacy.

As this was a cross-sectional analysis, recall bias could have been introduced, especially among fathers who have an older infant. In addition, fathers were recruited using convenience 
sampling. Thus, it may have contributed to study bias, especially since fathers who accompany their partners may have been more likely to actively participate in breastfeeding.

\section{CONCLUSION}

Multiple logistic regression analysis revealed three significant factors that influenced fathers' involvement in breastfeeding: fathers' breastfeeding knowledge, fathers' attitudes towards breastfeeding practices, and birth mode. These significant predictors may provide an insight to healthcare professionals to establish effective solutions and strategies for successful breastfeeding interventions and programmes involving fathers. Healthcare professionals and policy makers should implement strategies to engage fathers in the antenatal and breastfeeding classes to improve their overall knowledge and attitudes in breastfeeding in order to promote positive involvement of fathers in breastfeeding practices. Findings from this study may assist in the development of quantitative studies on a larger scale and with limited study bias. Future studies should also consider the effectiveness of fathers' involvement in breastfeeding and antenatal classes with breastfeeding outcomes.

\section{Acknowledgment}

This project was funded by the Lestari Research Grant (600-IRMI/DANA5/3/LESTARI(0101/2016) provided by the Institute of Research Management and Innovation, MARA University of Technology, Selangor, Malaysia. Special dedication to the staffs and participants attending the Kuala Selangor and Bestari Jaya Maternal and Child Primary Health Care facilities who have contributed directly and indirectly in the study.

\section{Authors' contributions}

NIMN conducted the study, led the data collection at selected primary healthcare facilities, data analysis and prepared the draft of the manuscript; SBAH is the principal investigator, conceptualised the study design, advised on data analysis and interpretation, and critically reviewed the final manuscript.

\section{Conflict of interest}

The authors have no conflict of interest to declare.

\section{References}

Abhinaya K, Arunprasath TS \& Padmasani LN (2016). Father's knowledge and attitude towards breastfeeding. Int $J$ Med Res Rev 4(10):1778-1785. https://doi.org/ doi:10.17511/ijmrr.2016.i10.12

Abu-Abbas MW, Kassab MI \& Shelash KI (2016). Fathers and breastfeeding process: determining their role and attitudes. Eur Sci $J$ 12(18):327-336.

Abdul Hamid SB \& Yahya N (2018). Knowledge, attitude, prior exposure and intention to breastfeed among undergraduate university students. Journal of Clinical and Health Sciences 3(2):26-35.

Al-Namir HMA, Brady AM \& Gallagher L (2017). Fathers and breastfeeding: Attitudes, involvement and support. $\mathrm{Br} J$ Midwifery 25(7):426-440.

Amin RM, Said ZM, Sutan R, Shah SA, Darus A \& Shamsuddin K (2011). Work related determinants of breastfeeding discontinuation among employed mothers in Malaysia. Int Breastfeed $J$ 6(1):2-7.

Bich T \& Cuong N (2016). Changes in knowledge, attitude and involvement of fathers in supporting exclusive breastfeeding: a community-based intervention study in a rural area of Vietnam. Int $J$ Public Health 62(1):1726.

Binns CW, Hauck YL, Maycock B, Burns S, Howat P \& Tohotoa J (2009). Dads make a difference: an exploratory study of paternal support for breastfeeding in Perth, Western Australia. Int Breastfeed $J$ 4(1):15.

Cox KN, Giglia RC \& Binns CW (2015). The influence of infant feeding attitudes on breastfeeding duration: evidence from a cohort study in rural Western Australia. Int Breastfeed J. 10:25.

de la Mora A, Russell DW, Dungy CI, Losch M \& Dusdieker L (2006). The Iowa Infant Feeding Attitude Scale: Analysis of reliability and validity. J Appl Soc Psychol 29(11):2362-2380.

de Montigny $\mathrm{F}$, Gervais $\mathrm{C}$, Larivière-Bastien D \& St-Arneault K (2018). The role of fathers during breastfeeding. Midwifery 58:6-12. 
dos Santos GM, dos S Albergaria TF, Lima FT, Santana AC \& Silva LR (2016). The knowledge and participation of the father in breast feeding, Salvador, Brazil. Open J Obstet Gynecol 06(10):559-571.

Draman N, Mohamad N, Yusoff HM \& Muhamad R (2017). The decision of breastfeeding practices among parents attending primary health care facilities in suburban Malaysia. J Taibah Univ Med Sci 12(5):412-417.

Hobbs AJ, Mannion CA, Mcdonald SW, Brockway M \& Tough SC (2016). The impact of caesarean section on breastfeeding initiation, duration and difficulties in the first four months postpartum. BMC Pregnancy Childbirth 16(90):1-9.

Laanterä S, Pietilä AM \& Pölkki T (2010). Knowledge of breastfeeding among pregnant mothers and fathers. J Perinat Neonatal Nurs 24(4):320-329.

Leahy-warren P, Mulcahy H, Phelan A \& Corcoran $P$ (2014). Factors influencing initiation and duration of breast feeding in Ireland. Midwifery 30(3):345-352.

Leng RNW, Shorey S, Yin SLK, Chan CPP \& He HG (2019). Fathers ' Involvement in Their wives '/ partners ' breastfeeding: a descriptive correlational study. J Hum Lact 35(4):801-812.

Lessen R \& Kavanagh K (2015). Position of the academy of nutrition and dietetics: promoting and supporting breastfeeding. J Acad Nutr Diet. 115(3):444-9.

Lester A (2014). Paternal support for breastfeeding: A mixed methods study to identify positive and negative forms of paternal social support for breastfeeding as perceived by first- time parent couples (Unpublished master's thesis). University of South Florida.

Maycock B, Binns CW, Dhaliwal S, Tohotoa J, Hauck Y, Burns S \& Howat P (2015). Education and support for fathers improves breastfeeding rates : a randomized controlled trial. J Hum Lact 29(4):484-490.

Mitchell-Box K, Braun KL, Hurwitz EL \& Hayes DK (2013). Breastfeeding attitudes: association between maternal and male partner attitudes and breastfeeding intent. Breastfeed Med 8(4):368-373. bfm.2012.0135

Mohamad N, Draman N, Muhamad R \& Yusof $\mathrm{H}$ (2015). Knowledge and attitude towards exclusive breastfeeding practices among fathers who attend Primary Health Care facilities in Suburban, Malaysia. Int $J$ Collab Res Intern Med Public Health 7(7):154-163.
Ng RWL, Shorey S \& He HG (2019). Integrative review of the factors that influence fathers' involvement in the breastfeeding of their infants. $J$ Obstet Gynecol Neonatal Nurs 48(1):16-26.

NCCFN (2016). National Plan of Action for Nutrition of Malaysia (NPANM III) 2016-2025. National Coordinating Committee on Food and Nutrition, Putrajaya, Malaysia.

IPH (2016). National Health and Morbidity Survey 2016 (NHMS 2016): Maternal and Child Health. Institute for Public Health, Ministry of Health Malaysia, Kuala Lumpur.

Phua HW, Razak NAA \& Shukri NHM (2020). Associations of father's breastfeeding attitude and support with the duration of exclusive breastfeeding among first-time mothers. Malaysian J Med Health Sci 16(Sup6):84-89.

Rempel LA, Rempel JK \& Moore KCJ (2017). Relationships between types of father breastfeeding support and breastfeeding outcomes. Matern Child Nutr 13(3):e12337. https://doi.org/10.1111/mcn.12337

Sherriff N, Hall V \& Panton C (2014). Engaging and supporting fathers to promote breast feeding: A concept analysis. Midwifery 30(6):667-677.

Shukri N, Wells J, Mukhtar F, Lee M \& Fewtrell M (2017). Study protocol: An investigation of mother-infant signalling during breastfeeding using a randomised trial to test the effectiveness of breastfeeding relaxation therapy on maternal psychological state, breast milk production and infant behaviour and growth. Int Breastfeed $J$ 12:33. https://doi.org/10.1186/s13006-0170124-y

WHO \& UNICEF (2014). Global Nutrition Targets 2025: Breastfeeding Policy Brief. World Health Organization, Geneva.

Zhao J, Zhao Y, Du M, Binns CW \& Lee AH (2017). Does caesarean section affect breastfeeding practices in China? a systematic review and meta-analysis. Matern Child Health $J$ 21(11):2008-2024. 